\title{
Cirugía mayor ambulatoria de la glándula tiroides y paratiroides. Resultados tras 6 años de experiencia
}

\author{
Alejandra de Andrés Gómez ${ }^{1}$, Francisco Leo Villalba Ferrer ${ }^{1}$, Antonio Melero Abellán ${ }^{1}$, \\ Ali Ismail ${ }^{1}$, Ramón Gómez Contreras ${ }^{1}$, Carla Navarro Moratalla ${ }^{1}$, \\ Javier Precioso Estiguín ${ }^{1}$ y Cristóbal Zaragoza Fernández ${ }^{1}$
}

Major outpatient surgery of the thyroid and parathyroid glands. Results after 6 years experience

Objetive: Ambulatory or overnight stay surgery have been consolidated in many different procedures. However, its use in thyroid and parathyroid surgery is still controversial. The aim of this report is to present the results of 6 years of ambulatory patients undergoing surgery of the thyroid or parathyroid glands. Material and Methods: Prospective observational study of patients who underwent hemithyroidectomy or selective parathyroidectomy in the ambulatory program from January 2011 to December 2016. All patients included met the general criteria of ambulatory surgery. During the first years nodules bigger than $3 \mathrm{~cm}$ and patients classified as ASA III were excluded. Patients arrive at hospital the morning of surgery. After the operation, patients pass to the post-anesthesia care unit and then to the hospitalization room. 8 hours after surgery patients are discharged home if they meet the criteria. Results: 270 patients were operated, 159 of them met the inclusion criteria. The overall applicability rate was $59 \%$. The acceptance rate was $83.6 \%$ and the substitution index was $49.2 \%$. The unwanted hospital admission was $10.4 \%$ for the parathyroidectomy and $17.6 \%$ for the hemithyroidectomy. Any patient presented major complications at home. The satisfaction rate was high or very high for $94 \%$ of the patients. Conclusion: Selective parathyroidectomy and hemithyroidectomy performed by experienced surgeons in selected patients can be safely and effectively carried out in ambulatory surgery (outpatient). It would be possible to improve the substitution index by increasing the application and acceptability ratios.

Key words: hemithyroidectomy; focused parathyroidectomy; ambulatory surgery; nodular goiter; primary hyperparathyroidism.

\section{Resumen}

Objetivo: La cirugía mayor ambulatoria (CMA) y con estancia inferior a 24 horas (overnight stay) se ha consolidado en muchas patologías aunque en cirugía tiroidea y paratiroidea resulta controvertido. El objetivo es valorar nuestros resultados en cirugía del tiroides y paratiroides en régimen de CMA durante un periodo de 6 años. Material y Métodos: Estudio observacional prospectivo de pacientes con indicación de hemitiroidectomía o paratiroidectomía enfocada en régimen CMA entre enero de 2011 y diciembre de 2016. Los pacientes cumplían criterios de CMA. En los primeros años se excluyeron los nódulos tiroideos mayores de $3 \mathrm{~cm}$ y los pacientes ASA III. Los pacientes ingresaron la mañana de la intervención, tras el acto quirúrgico pasaron a la Unidad de Recuperación Posquirúrgica y posteriormente a sala de hospitalización. Tras 8 h, si cumplían criterios, fueron dados de alta. Resultados: Se intervinieron 270 pacientes, la tasa de aplicabilidad global fue del 59\%, la tasa de aceptabilidad global del 83,6\% y el índice de sustitución del 49,2\%. El índice de ingresos no deseados fue del 10,4\% para la paratiroidectomía y del $17,6 \%$ en la cirugía del tiroides. Ningún paciente presentó complicaciones mayores en su domicilio. El grado de satisfacción fue alto o muy alto en el 94\% de los pacientes. Conclusiones: La paratiroidectomía enfocada y la hemitiroidectomía realizada por cirujanos expertos en pacientes seleccionados, es segura y efectiva en régimen ambulatorio. Es posible mejorar el índice de sustitución ambulatorio aumentando la tasa de aplicabilidad y aceptabilidad.

Palabras clave: hemitiroidectomía; paratiroidectomía enfocada; cirugía mayor ambulatoria; bocio nodular; hiperparatiroidismo primario. 


\section{Introducción}

La cirugía mayor ambulatoria (CMA) y con estancia inferior a $24 \mathrm{~h}$ (overnight stay) se ha consolidado en múltiples patologías tras demostrar que son coste-efectivas manteniendo la calidad asistencial y la seguridad del paciente, lo que ha propiciado su aplicación en muchas patologías ${ }^{1-4}$. Este régimen requiere una selección cuidadosa de pacientes y una continua evaluación de los resultados para su mejora $^{5}$.

Tradicionalmente la cirugía del tiroides y paratiroides presentaba estancias hospitalarias que en ocasiones eran superiores a 3 días $^{6}$. La aplicación de criterios de selección, el uso de nuevas tecnologías (nuevas energías, gafas lupa, neuromodulación, PTH intraoperatoria) y sobre todo la especialización de los profesionales en esta patología (cirugía, anestesia, neurofisiología, enfermería) han permitido la reducción de las estancias hospitalarias posoperatorias?.

Actualmente, los pacientes con patología tiroidea y paratiroidea que cumplen criterios de CMA, pueden ser incluidos en programas de Cirugía de Corta Estancia y $\mathrm{CMA}^{8,9}$. Sin embargo, la aplicación de este régimen ambulatorio ha sido muy controvertida por las complicaciones asociadas a estos procedimientos, fundamentalmente, el hematoma asfíctico, la lesión recurrencial y la hipocalcemia ${ }^{8,10}$. Por ello, la práctica de cirugía tiroidea y paratiroidea en régimen de CMA no se ha generalizado tanto como en otras patologías.

El objetivo de este trabajo es valorar nuestros resultados en la cirugía del tiroides y paratiroides en régimen de CMA durante un periodo de 6 años.

\section{Material y Métodos}

Estudio observacional prospectivo de los pacientes intervenidos en régimen de CMA por la Unidad de Cirugía Endocrina del Servicio de Cirugía General y del Aparato Digestivo del Hospital General Universitario de Valencia entre enero de 2011 y diciembre de 2016.

Los pacientes seleccionados cumplían criterios de inclusión para CMA en el hiperparatiroidismo primario por adenoma solitario e indicación de paratiroidectomía enfocada (HPTP-PTE) y el bocio nodular con indicación de hemitiroidectomía (BN-HT) (Tabla 1). Hasta diciembre de 2013 se excluyeron nódulos $>3 \mathrm{~cm}$ y pacientes ASA III, que posteriormente y hasta la actualidad dejaron de ser excluidos.

Todos los pacientes fueron informados, firmaron el consentimiento específico de CMA y recibieron un folleto informativo con las recomendaciones pre y posoperatorias (Anexos 1 y 2).

Se realizó ecografía cervical, punción con aspi-

Tabla 1. Criterios de inclusión/exclusión de pacientes

\begin{tabular}{|c|c|c|}
\hline & Criterios de inclusión & Criterios de exclusión \\
\hline Anestésicos & ASA I-II o III estable & ASA III inestable o ASA IV-V \\
\hline Quirúrgicos general & $\begin{array}{l}\text { No cirugía cervical previa } \\
\text { No antiagregantes/anticoagulantes }\end{array}$ & $\begin{array}{l}\text { Hemodiálisis } \\
\text { Sí antiagregantes/anticoagulantes } \\
\text { Embarazo } \\
\text { IMC }>40\end{array}$ \\
\hline Específicos tiroides & $\begin{array}{l}\text { Nódulo tiroideo único o múltiple subsidiario de hemitiroidectomía } \\
\text { Nódulo no tóxico } \\
\text { PAAF negativa de malignidad } \\
\text { No extemporánea }\end{array}$ & $\begin{array}{l}\text { Apnea del sueño } \\
\text { Trastorno convulsivo } \\
\text { Parálisis recurrencial unilateral } \\
\text { Tirotoxicosis }\end{array}$ \\
\hline Específicos paratiroides & $\begin{array}{l}\text { HPTP con adenoma único } \\
\text { Ausencia de HPT secundario o terciario } \\
\text { No enfermedad tiroidea asociada } \\
\text { No antecedente de HPT familiar o MEN } \\
\text { FA }<300 \mathrm{U} / \mathrm{L}\end{array}$ & $\begin{array}{l}\text { Apnea del sueño } \\
\text { Trastorno convulsivo } \\
\text { Parálisis recurrencial unilateral } \\
\text { Tirotoxicosis }\end{array}$ \\
\hline
\end{tabular}


ración con aguja fina (PAAF) y estudio analítico a todos los pacientes con patología tiroidea y se añadió la gammagrafía con sestamibi en los HPTP-PTE.

En los pacientes intervenidos por HPTP-PTE, se realizó determinación intraoperatoria de PTH sanguínea siguiendo criterio de curación de Miami y estudio anatomopatológico intraoperatorio para confirmar tejido paratiroideo.

Los pacientes ingresaron el mismo día de la intervención, y ocuparon el primer lugar del parte quirúrgico matinal. Tras la cirugía pasaron a la sala de recuperación posquirúrgica durante $2 \mathrm{~h}$ y posteriormente a la sala de cirugía donde permanecieron con un familiar.

Tras $8 \mathrm{~h}$ de posoperatorio el paciente fue valorado por el cirujano y dado de alta si cumplía los criterios (Tabla 2).

$\mathrm{Al}$ alta, se entregó folleto informativo e informe de alta con tratamiento posoperatorio, cuidados de la herida y actitud a seguir ante posibles complicaciones, así como una encuesta de satisfacción (grado de satisfacción muy alto, alto, medio o bajo). Se les facilitó un número de teléfono de contacto y se les instruyó para que acudiesen a urgencias en caso de complicaciones.

Se estableció control posoperatorio con llamada telefónica a las $24 \mathrm{~h}$ y revisión en consultas externas al mes de la intervención.

\section{Técnica anestésica y quirúrgica}

Durante la anestesia de ambos procesos se realizó profilaxis antiemética con 4-8 mg de dexametasona tras inducción y $4 \mathrm{mg}$ de ondansetrón al final de la cirugía. Como profilaxis de la tos, se administró

Tabla 2. Criterios que deben cumplirse en el momento del alta

\begin{tabular}{|l|}
\hline - Signos vitales y tensión arterial estables \\
- No equimosis, hematomas ni tumefacción cervical \\
- Drenaje no productivo \\
- No disfonía, disnea o disfagia \\
- Buena tolerancia oral para líquidos y sólidos \\
- Control adecuado del dolor con tratamiento oral \\
- Deambulación correcta \\
- Comprensión de las instrucciones \\
- Capacidad para el cuidado personal \\
- Adecuado soporte domiciliario \\
- Posibilidad de control telefónico
\end{tabular}

lidocaína i.v. y tópica y se realizó la extubación del paciente durante el plano profundo de anestesia.

En el BN-HT se restringió el uso de relajantes musculares para el control recurrencial mediante neuromonitorización.

El acceso al tiroides se realizó con incisiones mínimas centrales, de $3 \mathrm{~cm}$ (ampliable según hallazgos), $2 \mathrm{~cm}$ por encima del hueco esternal y separación lateral de musculatura pretiroidea.

Se emplearon gafas lupa (Eyezoom optimedic ${ }^{\circledR}$ ), bisturí monopolar, energía ultrasónica (Harmonic focus ${ }^{\circledR)}$ y clips metálicos en la zona peri-recurrencial. Se siguieron los mismos pasos de resección tiroidea estándares que en cirugía convencional, con identificación sistemática de los nervios vago, recurrente y valoración funcional de ambos con neuromonitorización. La neuromonitorización se realizó con estímulo intermitente mediante sistema Medtronic Nim-Neuro 3.0® los primeros 4 años. En los últimos 2 años se incorporó la neuromonitorización continua realizada por el neurofisiólogo del hospital con electrodos anexados al tubo endotraqueal y electrodo de neuroestimulación continua sobre el nervio vago (inomedV3 Vagus Electrode ${ }^{\circledR}$ ).

En la cirugía del HPTP-PTE el abordaje se realizó en función de la concordancia o no de las pruebas de imagen (ecografía y gammagrafía con setamibi).

En caso de concordancia de imágenes se realizó un abordaje lateral con incisión mínima ampliable de $2 \mathrm{~cm}$ sobre la glándula patológica y accediendo entre musculatura pretiroidea y esternocleidomastoideo.

Cuando no existía concordancia de imágenes se realizó abordaje medial con incisión mínima ampliable de $3 \mathrm{~cm}$ al igual que en la cirugía tiroidea con exploración en primer lugar de la localización de la imagen patológica gammagráfica. No se realizó neuromonitorización.

\section{Análisis estadístico}

El análisis se ha realizado en función de la naturaleza de sus variables. Para variables cualitativas se han calculado frecuencias (absolutas y relativas) y para variables cuantitativas, se han calculado medias y desviaciones estándar, tras comprobar su distribución normal mediante el test de KolmogorovSmirnov.

Las diferencias observadas entre las medias se han analizado con la $t$ de student para muestras relacionadas y test paramétrico para variables dependientes que siguen una distribución normal.

Un valor $\mathrm{p}<0,05$ ha sido considerado estadísticamente significativo. Los datos se han analizado mediante SPSS Inc., versión 23 para Windows. 
Tabla 3. Aplicabilidad de régimen de cma

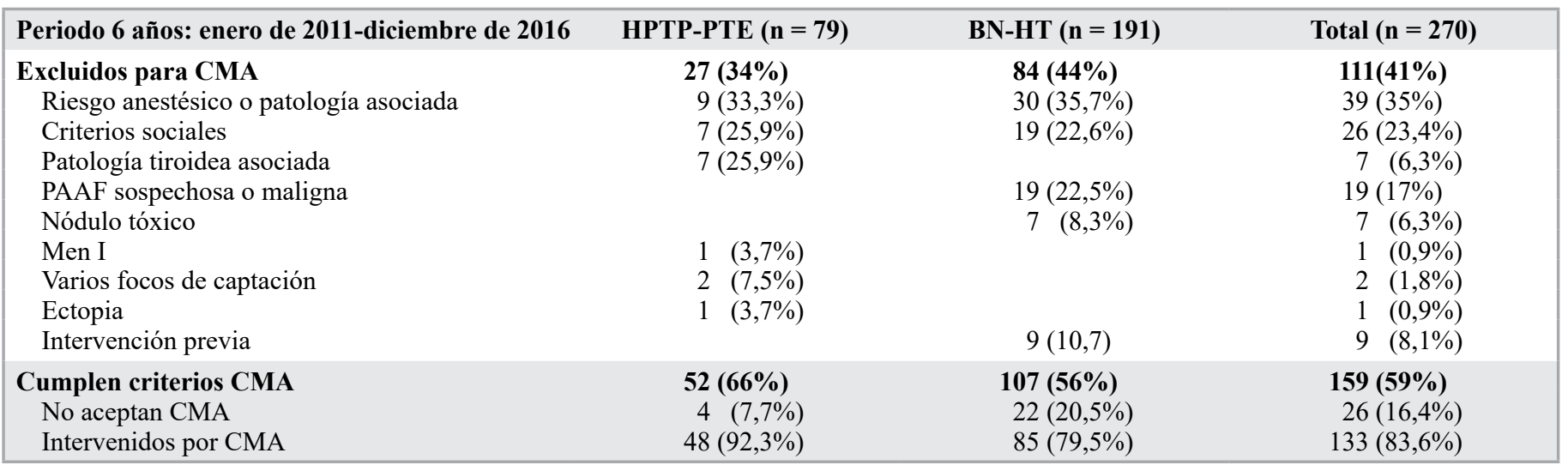

HPTP-PTE: hiperparatiroidismo primario con indicación de paratiroidectomía enfocada; BN-HT: bocio nodular con indicación de hemitiroidectomía; CMA; cirugía mayor ambulatoria.

Tabla 4. Características epidemiológicas de los pacientes intervenidos por CMA

\begin{tabular}{|lccc|}
\hline & HPTP-PTe $(\mathbf{n}=\mathbf{4 8})$ & BN-HT $(\mathbf{n}=\mathbf{8 5})$ & Total $(\mathbf{n}=\mathbf{1 3 3})$ \\
Edad media & $60 \pm 13,3$ & $46,2 \pm 12,3$ & $53 \pm 13$ \\
Sexo & & & \\
$\quad$ Hombre & $8(16,7 \%)$ & $15(17,6 \%)$ & $23(17,3 \%)$ \\
Mujer & $40(83,3 \%)$ & $70(82,4 \%)$ & $110(82,7 \%)$ \\
Grado ASA & & & \\
ASA I & $18(37,5 \%)$ & $36(42,3 \%)$ & $54(40,6 \%)$ \\
ASA II & $26(54,2 \%)$ & $44(51,8 \%)$ & $70(52,6 \%)$ \\
ASA III & $4(8,3 \%)$ & $5(5,9 \%)$ & $9(6,8 \%)$ \\
\hline
\end{tabular}

HPTP-PTE: hiperparatiroidismo primario con indicación de paratiroidectomía enfocada; BN-HT: bocio nodular con indicación de hemitiroidectomía; ASA: American Society of Anesthesiologists

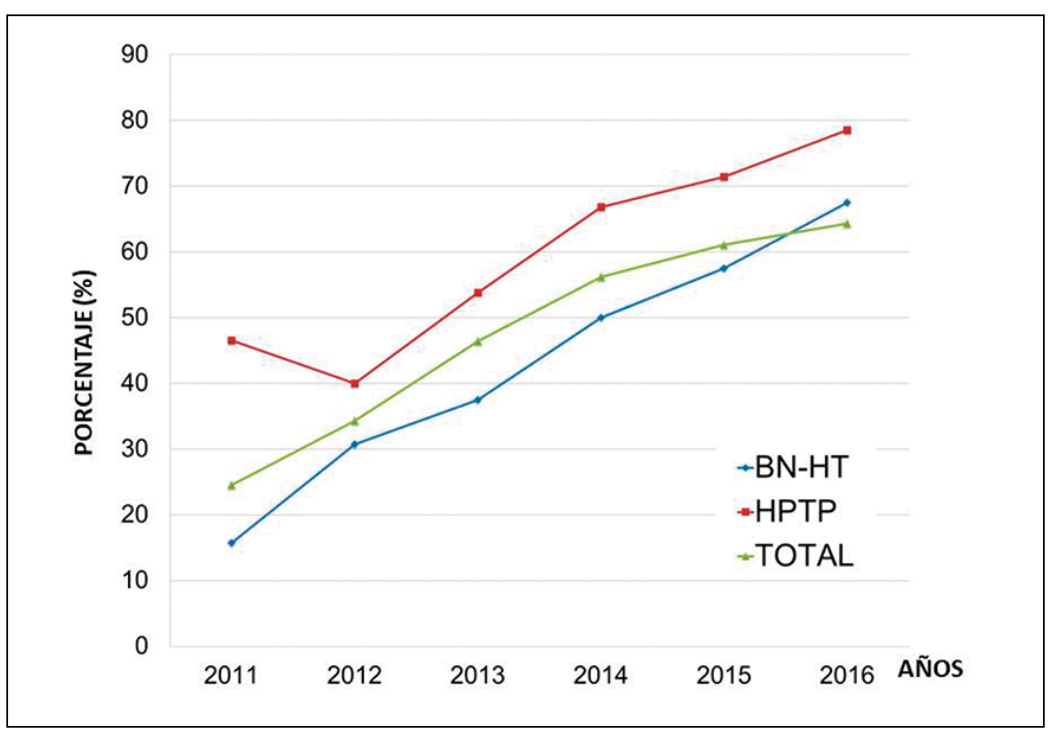

Figura 1. Índice de sustitución. HPTP-PTE: hiperparatiroidismo primario con indicación de paratiroidectomía enfocada; BN-HT: bocio nodular con indicación de hemitiroidectomía.

\section{Resultados}

Durante el periodo de 6 años (enero de 2011-diciembre de 2016) hemos intervenido un total de 270 pacientes, 79 con HPTP-PTE y 191 pacientes con BN-HT.

Cumplían criterios de inclusión en CMA 159 pacientes lo que supone una tasa de aplicabilidad global del protocolo de inclusión del $59 \%, 52$ pacientes (66\%) en el HPTP-PTE y 107 (56\%) en el BN-HT.

No cumplieron criterios de inclusión 111 pacientes $(41 \%)$ y no aceptaron el régimen de CMA a pesar de cumplir criterios $26(16,4 \%)$, lo que supone una tasa de aceptabilidad global del 83,6\% (HPTPPTE: $92,3 \%$ y BN-HT:79,5\%) (Tabla 3).

Un total de 133 pacientes fueron intervenidos en régimen de CMA (48 HPTP-PTE y $85 \mathrm{BN}-\mathrm{HT}$ ). La edad media fue de $53 \pm 13$ años con predominio del sexo femenino $(82,7 \%)$ (Tabla 4$)$.

En todos los casos se trataba de pacientes ASA I, II o III estables. El índice de sustitución global ha sido del 49,2\% (133/270), con aumento significativo $(\mathrm{p}<0,05)$ desde el año $2011(24,5 \%)$ hasta el año $2016(64,3 \%)$ (Figura 1).

En la cirugía del BN-HT el tamaño ecográfico del nódulo dominante fue de $3 \pm 1,5 \mathrm{~cm}$. Todos los pacientes incluidos fueron eutiroideos. Se realizó hemitiroidectomía a todos los pacientes con un tiempo operatorio de $65 \pm 15 \mathrm{~min}$. El tamaño medio de la incisión fue de $3,8 \pm 0,7 \mathrm{~cm}$. Se realizó neuromonitorización en todas las cirugías de BN-HT sin pérdida de señal en ningún caso.

En el HPTP-PTE presentaron concordancia de imagen 36 pacientes $(75 \%)$. Se realizaron 37 abordajes laterales $(77 \%)$ con mini-incisión enfo- 
cada $(2,5 \pm 0,7 \mathrm{~cm})$ sobre la glándula patológica, 36 pacientes con imágenes concordantes y uno sin concordancia. En 11 pacientes sin concordancia de imágenes se realizaron abordajes centrales $(23 \%)$ con mini-incisión central de Kocher de $(3 \pm 0,7 \mathrm{~cm})$.

De los 133 pacientes intervenidos por CMA, 113 $(81,5 \%)$ fueron dados de alta $8 \mathrm{~h}$ tras la cirugía y permanecieron ingresados 20 pacientes $(18,5 \%)$ que fueron dados de alta al día siguiente (Tabla 5).

El índice de ingresos no deseados para BN-HT fue del 17,6\% (15 pacientes): 2 por tos, 1 presentó vómitos posoperatorios, 3 disfonía, 1 presentó crisis epiléptica posoperatoria y 8 pacientes no aceptaron el alta en el mismo día a pesar de no presentar complicaciones

El índice de ingresos no deseados para el HPTPPTE fue del 10,4\% (5 pacientes): 1 por vómitos, 2 por prolongación de la cirugía y 2 que no aceptaron el alta a pesar de no tener complicaciones. La prolongación de la cirugía fue por un hallazgo hiperplasia y por una ectopia no localizada con las pruebas de imagen ni durante la intervención (Tabla 5).

Hubo un reingreso a las $48 \mathrm{~h}$ por hipocalcemia sintomática de un paciente del grupo HPTP-PTE. No se presentó ninguna hemorragia posoperatoria ni complicaciones de herida.

En el seguimiento se detectó disfonía transitoria en tres pacientes intervenidos de hemitiroidectomía. En todos los pacientes con disfonía se realizó laringoscopia posoperatoria que resultó normal.

En el grupo de HPTP-PTE las cifras de $\mathrm{Ca}$ y PTH fueron normales en 36 pacientes al mes, 3 y 6 meses. En el paciente con ectopia no localizada persistió elevación del Ca y PTH siendo diagnosticado a los 6 meses con nueva gammagrafía y TC cérvico-torácico de adenoma ectópico en mediastino que requirió intervención.

El grado de satisfacción fue alto o muy alto en el 94\% de los pacientes (Tabla 6).

\section{Discusión}

Hemos conseguido una tasa de aplicabilidad para CMA del 59\%, inferior a la de otras series $(75,8 \%)^{8}$, posiblemente porque al inicio del programa fuimos muy restrictivos en la selección de los pacientes, sobre todo en los pacientes con BN-HT.

Nuestra tasa de aceptación para CMA, en los pacientes que cumplían criterios de HPTP-PTE, ha sido superior a la de otras series ${ }^{8}$, posiblemente porque planteaban la cirugía con anestesia local y hubo mayor tasa de renuncias.
Tabla 5. Ingresos no deseados

\begin{tabular}{|lccc|}
\hline Motivo de ingreso & $\begin{array}{c}\text { HPTP-PTE } \\
\mathbf{n}=\mathbf{5}\end{array}$ & $\begin{array}{c}\text { BN-HT } \\
\mathbf{n}=\mathbf{1 5}\end{array}$ & $\begin{array}{c}\text { Total } \\
\mathbf{n}=\mathbf{2 0}\end{array}$ \\
\hline Tos & 0 & $2(13,3 \%)$ & $2(10 \%)$ \\
Vómitos & $1(20 \%)$ & $1(7,7 \%)$ & $2(10 \%)$ \\
Disfonía & 0 & $3(20 \%)$ & $3(15 \%)$ \\
Prolongación cirugía & $2(40 \%)$ & 0 & $2(10 \%)$ \\
Crisis epiléptica & 0 & $1(7,7 \%)$ & $1(5 \%)$ \\
No aceptación alta & $2(40 \%)$ & $8(53,3 \%)$ & $10(50 \%)$ \\
Total serie & $5 / 48(10,4 \%)$ & $15 / 85(17,6 \%)$ & $18 / 133(13,5 \%)$ \\
\hline
\end{tabular}

HPTP-PTE: hiperparatiroidismo primario con indicación de paratiroidectomía enfocada; BN-HT: bocio nodular con indicación de hemitiroidectomía.

Tabla 6. Grado de satisfacción

\begin{tabular}{|l|c|}
\hline & n \\
\hline Muy alto & $85(64 \%)$ \\
\hline Alto & $40(30 \%)$ \\
\hline Medio & $4(3 \%)$ \\
\hline Bajo & $4(3 \%)$ \\
\hline
\end{tabular}

En la cirugía del BN-HT nuestra tasa de aceptabilidad es menor que la de otras series donde se alcanza hasta el $98 \%{ }^{11}$, posiblemente porque en la información preoperatoria no fuimos suficientemente convincentes, tal y como se destaca en otras publicaciones $^{7,11}$.

En el BN-HT nuestro índice de sustitución es bajo comparado con otras series ${ }^{11}$, debido a que inicialmente nuestros criterios de inclusión eran más restrictivos y hasta el 2013 no incluimos el ASA III estable ni los nódulos $>3 \mathrm{~cm}$. Posteriormente, detectamos un ascenso significativo del índice de sustitución sobre todo en los dos últimos años del estudio.

Hay que destacar la importancia de la especialización en la cirugía endocrina ${ }^{5,8,12,13}$ y sobre todo en la cirugía ambulatoria, donde la morbilidad debe ser mínima. Todos nuestros pacientes fueron intervenidos por cirujanos con dedicación exclusiva a la cirugía endocrina, con el apoyo de la nueva tecnología y la infraestructura del Servicio de CMA.

Es recomendable una estancia posoperatoria que garantice la seguridad del paciente, puesto que el periodo de mayor incidencia de los hematomas posquirúrgicos ocurre antes de las $6 \mathrm{~h}^{6}$. El ingreso de nuestros pacientes el mismo día de la cirugía y 
la intervención en primer lugar del parte quirúrgico permitió un período de recuperación de $2 \mathrm{~h}$ en la Unidad de Recuperación Posquirúrgica y 6 h en sala de hospitalización (mínimo de $8 \mathrm{~h}$ ).

La colaboración del anestesista es fundamental para el uso de la neuromonitorización y deben evitarse los relajantes neuromusculares puesto que impiden el registro de los potenciales evocados. En la inducción anestésica se pueden utilizar relajantes musculares de acción corta ${ }^{14}$. Todos los pacientes intervenidos de BN-HT recibieron anestesia con restricción de relajantes musculares y se realizó neuromonitorización en todos ellos sin tener problemas en los registros.

En la cirugía cervical es indispensable la profilaxis de la tos, las náuseas y los vómitos posoperatorios que causan disconfort, pueden retrasar el alta e incluso favorecer la hemorragia posoperatoria. A pesar, de las profilaxis realizadas en todos nuestros pacientes, 4 permanecieron ingresados por persistencia de nauseas, vómitos y tos.

La tasa de ingresos no deseados (15\%) fue elevada a pesar de la selección de pacientes, debido a los 10 pacientes que no quisieron el alta a pesar de no presentar complicaciones. Esto ocurrió mayoritariamente al inicio del programa ambulatorio, posiblemente, por no ser suficientemente explícitos con la información preoperatoria. Actualmente, empleamos más tiempo con el paciente en la consulta a fin de asegurar que entiende y acepta el circuito asistencial ambulatorio.

El principal obstáculo para llevar a cabo la ambulatorización de estos procesos es el sangrado posoperatorio. El sangrado tras la tiroidectomía en el posoperatorio inmediato tiene una incidencia entre el 0,5 y el $3 \%$ de los casos, siendo más frecuente en las primeras $6 \mathrm{~h}(75 \%)$ y excepcional después de las $24 \mathrm{~h}$ del posoperatorio ${ }^{6}$. Esta es la razón por la que algunos cirujanos endocrinos realizan la cirugía tiroidea en un día o con recuperación prolongada $(23,59 \mathrm{~h})^{15-17}$. En la cirugía de la glándula paratiroides, la incidencia de sangrado es inferior a la de la cirugía de la tiroides, siendo menor del 1\%, al no precisar la ligadura de pedículos vasculares importantes ni una gran disección de los planos. En caso de producirse, ocurre en el $90 \%$ de los casos durante la primera hora del posoperatorio, siendo en el $85 \%$ de los casos durante la extubación y las maniobras de Valsalva. Entre la primera y la sexta hora es del $10 \%$ y prácticamente inexistente a partir de la sexta hora posoperatoria ${ }^{18}$. En nuestra serie, con una estancia hospitalaria mínima de $8 \mathrm{~h}$ no hemos detectado ningún signo de alarma de sangrado y fueron dados de alta sin ningún reingreso por esta causa.
Otro de los inconvenientes que se atribuye al régimen ambulatorio en la cirugía tiroidea es el riesgo de una lesión recurrencial, sobre todo bilateral. Esta es la razón por la que no incluimos pacientes con indicación de tiroidectomía total o intervención previa. En la hemitiroidectomía no existe posibilidad de lesión recurrencial bilateral y además, utilizamos la neuromonitorización para detectar la lesión nerviosa ${ }^{16} \mathrm{y}$, en tal caso, a pesar de ser unilateral lo recomendable es ingresar al paciente. Sin el uso de la neuromonitorización pueden pasar desapercibidas lesiones funcionales recurrenciales que en caso de sangrado agravarían un cuadro de asfixia. En nuestra serie 3 pacientes con BUN-HT presentaron disfonía posoperatoria sin asociarse a lesión recurrencial mediante neuroestimulación. A pesar de la integridad recurrencial, los pacientes quedaron ingresados como precaución a un sangrado asociado que pudiera agravar un cuadro de lesión funcional recurrencial no diagnosticado.

Otra de las complicaciones en la cirugía del tiroides y paratiroides es el hipoparatiroidismo. Esta complicación no se presenta en la cirugía del $\mathrm{BN}-\mathrm{HT}$, puesto que se realiza un abordaje unilateral quedando intactas paratiroides contralaterales, motivo por el cual la tiroidectomía total ha sido uno de nuestros criterios de exclusión.

En la cirugía del HPTP-PTE tampoco se produce hipocalcemia permanente puesto que no se manipulan las glándulas contralaterales, aunque sí podría ocurrir un hipoparatiroidismo transitorio ${ }^{19}$. En nuestra serie, tuvimos 2 casos de hipocalcemia posoperatoria transitoria y sólo una de ellas fue sintomática y requirió reingreso. Por tanto, aunque un porcentaje de pacientes pueden presentar una hipocalcemia leve $(7-8 \mathrm{mg} / \mathrm{dl})$ transitoria, ésta no produce habitualmente sintomatología clínica $o$, en caso de producirse, se soluciona con tratamiento médico y no supone ningún riesgo para el paciente.

\section{Conclusiones}

La cirugía del adenoma solitario de paratiroides con indicación de paratiroidectomía enfocada y del bocio nodular con indicación de hemitiroidectomía en régimen ambulatorio, es segura y efectiva en pacientes bien seleccionados, realizada por cirujanos especializados y con apoyo de las nuevas tecnologías.

Es posible mejorar el índice de sustitución ambulatorio aumentando la tasa de aplicabilidad y aceptabilidad. 


\section{Responsabilidades éticas}

Protección de personas y animales. Los autores declaran que para esta investigación no se han realizado experimentos en seres humanos ni en animales.

Confidencialidad de los datos. Los autores declaran que en este artículo no aparecen datos de pacientes.

Derecho a la privacidad y consentimiento informado. Los autores declaran que en este artículo no aparecen datos de pacientes.

Conflictos de interés: no hay.

\begin{tabular}{|l|}
\hline Anexo 1. Recomendaciones generales preoperatorias \\
\hline - La noche antes de la intervención realice una cena ligera, a partir de entonces permanezca en ayunas \\
\hline - Tomar la medicación prescrita \\
\hline - Duchar en la mañana de la intervención \\
\hline - El día de la intervención no desayune ni tome nada por boca (excepto que se le haya prescrito medicación) \\
\hline - Si utiliza prótesis dental o lentillas, retírelas antes de venir al Servicio \\
\hline - Vista ropa cómoda, sin joyas, maquillaje o pintura de uñas \\
\hline - Traiga su medicación habitual \\
\hline - Si es diabético no se pinche insulina ni tome pastillas para la diabetes \\
\hline - Venga acompañado/a de un adulto responsable \\
\hline - Usted no puede conducir ni antes ni después de la intervención \\
\hline
\end{tabular}

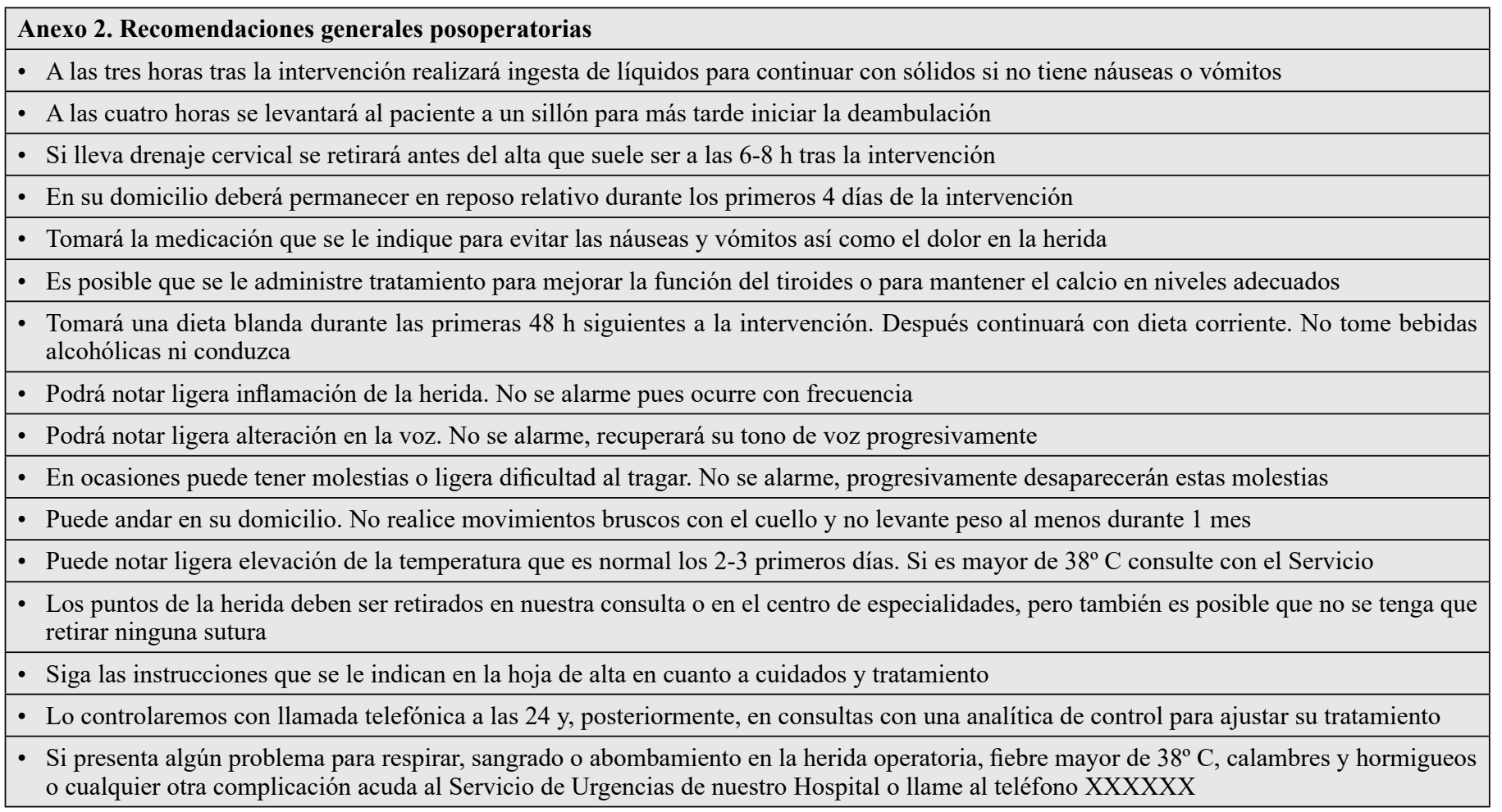




\section{Referencias}

1. Martí Sánchez J, Prats Maeso M, Barja Sánchez J, Cruz Verdún J, Hidalgo Grau LA, Suñol Sala X. Resultados iniciales de un programa de cirugía tiroidea sin ingreso en un hospital general. Cir May Amb. 2013;18:97-101.

2. Guerrero AM. Estructura y funcionamiento de la Cirugía Mayor Ambulatoria. En: Ruiz P, Alcalde J, Landa JI. Gestión Clínica en Cirugía. Madrid: Aran Ediciones; 2005. p. 215-32.

3. Mirnezami R, Sahai A, Symes A, Jeddy T. Day-case and short-stay surgery: the future for thyroidectomy? Int J Clin Pract. 2007;61:1216-22.

4. Martínez Ramos C, Sanz-López R. Ambulatorización de la cirugía tiroidea Cir May Amb. 2007;12:4-9.

5. Sánchez-Blanco JM, Díaz-Roldán J, Guerola-Delgado A. Paratiroidectomía ambulatoria bajo anestesia local tras exploración cervical bilateral en el hiperparatiroidismo primario. Estudio Prospectivo. Cir Esp. 2004;76:213-8.

6. Schwartz AE, Clark $\mathrm{OH}$, Ituarte $\mathrm{P}$, Lo Gerfo P. Therapeutic controversy: Thyroid surgery-the choice. J Clin Endocrinol Metab. 1998;83:1097-105.
7. Moreno P. Cirugía endocrina en régimen ambulatorio (CMA). Cir Esp. 2006;80:273-4.

8. Sánchez-Blanco JM. Cirugía tiroidea y paratiroidea en régimen ambulatorio. En Cirugía Endocrina. Guía Clínica de la Asociación Española de Cirujanos. 2 ${ }^{\mathrm{a}}$ edición. Capítulo 14. Aran ed. 2009.

9. Steckler RM. Outpatient thyroidectomy: a feasibility study. Am J Surg. 1986;152:417-9.

10. Hopkins B, Steward D. Outpatient thyroid surgery and the advances making it possible. Curr Opin Otolaryngol Head Neck Surg. 2009; 17:95-9.

11. Godballe C, Madsen AR, Pedersen HB, Hjort C, Pedersen U, Frisch T, et al. Post-Thyroidectomy hemorrhage: a national study of patients treated at the Danish departments of ENT Head and Neck Surgery. Eur Arch Otorhinolaryngol. 2009;266:1945-52.

12. Sosa JA, Bowman HM, Tielsch JM, Powe NR, Gordon TA, Udelsman R. The importance of surgeon experience for clinical and economic outcomes from thyroidectomy. Ann Surg. 1998;228:32030.

13. Sherman SI. The risks of thyroidectomy.
Words of caution for referring physicians. J Gen Intern Med. 1998;13:60-1.

14. Deiner S. Highlights of anesthetic considerations for intraoperative neuromonitoring. Semin Cardiothorac Vasc Anesth. 2010;14:51-3.

15. Sahai A, Symes A, Jeddy T. Short-stay thyroid surgery. Br J Surg. 2005;92:58-9.

16. Sanabria A, Ramírez A, Kowalski LP, Silver CE, Shaha AR, Owen RP, et al. Neuromonitoring in thyroidectomy: A meta-analysis of effectiveness from randomized controlled trials. Eur Arch Otorhinolaryngol. 2013;270:2175-89.

17. Samson PS, Reyes FR, Saludares WN, Ángeles RP, Ricardo A, Tagorda ER. Outpatient thyroidectomy. Am J Surg. 1997;173:499-503.

18. Irvin III GL, Sfakianakis G, Yeung L, Deriso GT, Fishman LM, Molinari AS, et al. Ambulatory parathyroidectomy for primary hyperparathyroidism. Arch Surg. 1996;131:1074-8.

19. Van Vroonhoven TJ, van Dalen A. Successful minimally invasive surgery in primary hyperparathyroidism after combined preoperative ultrasound and computed tomography imaging. J Intern Med. 1998;243:581-7. 